\title{
Radial gradients in the SLUGGS survey
}

\section{Caroline Foster and the SLUGGS collaboration}

\author{
email: cfoster@aao.gov.au
}

\begin{abstract}
The SAGES Legacy Unifying Galaxies and GlobularS (SLUGGS) survey probes the outskirts of early-type galaxies (ETGs). SLUGGS uses the DEIMOS spectrograph on Keck to simultaneously obtain globular cluster and spatially resolved galaxy spectra. The galaxy spectra allow for the reconstruction of kinematic maps out to $\sim 3$ effective radii $\left(R_{e}\right)$, while globular clusters (GCs) push the galactocentric limit out to $\sim 8-10 R_{e}$. Stellar population parameters (abundances) can also be recovered. This provides a unique dataset for exploring the outer gradients (abundance and/or kinematic transition). We briefly present recent and salient results of the SLUGGS survey so far.
\end{abstract}

Keywords. galaxies: elliptical and lenticular - galaxies: kinematic - galaxies: abundances galaxies: formation

\section{Introduction}

The two-phase galaxy formation scenario has recently been proposed in order to explain the fast growth in size of massive ETGs since redshift $z \sim 2$ (e.g. Daddi et al. 2005). Under this scenario, massive galaxies form in two phases: 1) early central bulge formation via multiple major mergers or dissipative processes at high redshift (e.g. Ceverino et al. 2014) and 2) subsequent series of multiple minor merger events increasing the size by preferably adding stars to the outskirts (e.g. Naab et al. 2009; van Dokkum et al. 2010). Hence, these in-situ bulge and the ex-situ halo components should be readily detectable observationally. Such transitions are also predicted in other formation scenarios (e.g. Hoffman et al. 2009).

The SAGES Legacy Unifying Galaxies and GlobularS (SLUGGS) survey (Brodie et al. 2014) uses a combination of the Stellar Kinematics using Multiple Slits (SKiMS) technique (Proctor et al. 2009) and globular cluster data to push the galactocentric boundary beyond what is typically allowed by integral field spectrographs (IFSs) in order to probe the faint outskirts of local massive ETGs. The SLUGGS sample contains 25 ETGs (ellipticals and lenticulars) from a representative range of environments. Data for 3 "bonus" galaxies are also being gathered.

In this short contribution, we briefly present our data (Section 2) and highlight some recent results (Section 3).

\section{Data}

The SLUGGS data and products are described in detail in Brodie et al. (2014) and more information can be found on our website (http://sluggs.swin.edu.au/). Here we give a brief overview.

The SLUGGS survey comprises an imaging and a follow-up spectroscopic component. The imaging data combine pre-existing archival data from various sources with wide-field multi-band imaging using Subaru/Suprime-cam. Spectra are obtained using the DEIMOS spectrograph on Keck in multi-slit mode. The instrument setup allows coverage of the near-infrared Calcium II Triplet around $8500 \AA$. The obtained spectral resolution is 


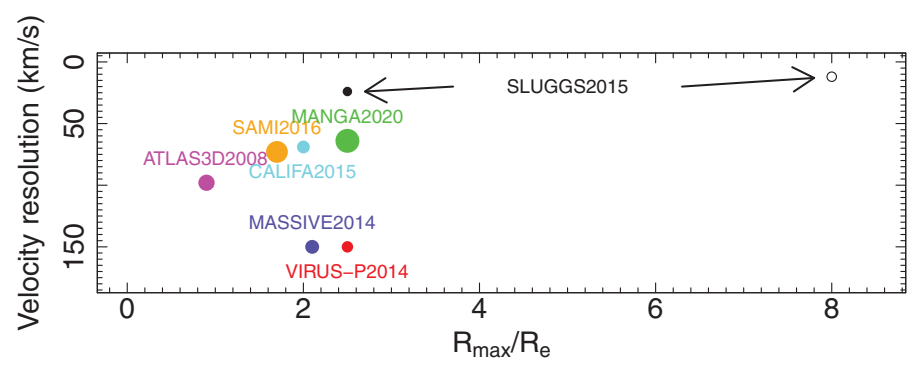

Figure 1. Comparison of the spatial extent and velocity resolution of SLUGGS with other past and current IFS surveys as labelled. The size of the symbol is proportional to the logarithm of the sample size. The GC and galaxy light components of SLUGGS are shown as hollow and filled black circles, respectively. The SLUGGS survey probes an area in this parameter space that was previously unexplored.

FWHM $\sim 1.5 \AA$, affording a velocity resolution of $\sim 24$ and $\sim 12 \mathrm{~km} \mathrm{~s}^{-1}$ for the galaxy and GC components of the survey, respectively. As a result, the SLUGGS survey probes a yet unexplored area of parameter space (see Fig. 1).

\section{Radial gradients}

Kinematic transitions have already been detected as part of SLUGGS (e.g. Foster et al. 2013; Arnold et al. 2014), thereby supporting the two-phase galaxy formation paradigm. In Pastorello et al. (2014), we find a remarkable trend in the outer stellar metallicity profiles such that massive galaxies have shallower outer profiles, indicating they have undergone a more active accretion history than low mass galaxies (e.g. White 1980). In parallel, Usher et al. (2012) and Pota et al. (2013) present the globular cluster abundances and kinematics, respectively, reaching out to several effective radii. The abundances and kinematics of SLUGGS galaxies are combined with that of their respective globular cluster systems in Pastorello et al. (in prep.) and Alabi et al. (in prep.), respectively.

\section{References}

Arnold J. A., et al. 2014, ApJ, 791, 80

Brodie J. P., et al. 2014, ApJ in press, arXiv:1405.2079

Ceverino D., Dekel A., Tweed D., \& Primack J., 2014, ArXiv e-prints

Daddi E., et al. 2005, ApJ, 626, 680

Foster C., Arnold J. A., Forbes D. A., Pastorello N., Romanowsky A. J., Spitler L. R., Strader J., \& Brodie J. P., 2013, MNRAS, 435, 3587

Hoffman L., Cox T. J., Dutta S., \& Hernquist L., 2009, ApJ, 705, 920

Naab T., Johansson P. H., \& Ostriker J. P., 2009, ApJ, 699, L178

Pastorello N., Forbes D. A., Foster C., Brodie J. P., Usher C., Romanowsky A. J., Strader J., \& Arnold J. A., 2014, MNRAS, 442, 1003

Pota V., et al. 2013, MNRAS, 428, 389

Proctor R. N., Forbes D. A., Romanowsky A. J., Brodie J. P., Strader J., Spolaor M., Mendel J. T., \& Spitler L., 2009, MNRAS, 398, 91

Usher C., et al. 2012, MNRAS, 426, 1475

van Dokkum P. G., et al. 2010, ApJ, 709, 1018

White S. D. M., 1980, MNRAS, 191, 1P 